\title{
Myeloid Sarcoma Transformed From CML Presenting Ectopic Expression of AFP: A Case Report
}

\section{Ying Wang}

Ruijin Hospital, Shanghai Jiao Tong University School of Medicine

Haimin Sun

Ruijin Hospital, Shanghai Jiao Tong University School of Medicine

Sujiang Zhang ( $\square$ zbruce.zhang@hotmail.com)

National Research Center for Translational Medicine at Shanghai, Ruijin Hospital『Shanghai Jiao Tong University School of Medicine

\section{Case Report}

Keywords: Myeloid Sarcoma, chronic myeloid leukemia, AFP, mutation, multiple osteopathy

Posted Date: February 23rd, 2022

DOI: https://doi.org/10.21203/rs.3.rs-1374914/v1

License: (c) (i) This work is licensed under a Creative Commons Attribution 4.0 International License. Read Full License 


\section{Abstract}

Background囚Myeloid Sarcoma (MS) may be transformed from chronic myeloid leukemia (CML). However, no any relationship about MS and significant elevated Alpha-fetoprotein (AFP) as well as multiple osteolytic damage was reported.

Case presentation囚A 38-years-old man, who was firstly diagnosed as CML chronic phase and treated with imatinib eight years ago, gradually progressed into MS with bone pain and multiple osteolytic damage, especially with elevated AFP. Immunohistochemical staining strongly confirmed that AFP is secreted by MS and its level is fluctuated with treatment outcome. Sequential compound ABL mutations were also identified in bone marrow and finally the patient lost any treatment response to targeted therapy as well as combined chemotherapy.

Discussion and Conclusions区To our knowledge, this case is the first report about MS with aberrant AFP expression.

\section{Background}

Myeloid Sarcoma (MS) is a tumoral lesion consisting of immature granulocytic cells and may arise de novo in isolation or accompany with acute myeloid leukemia. Less frequently associations with myeloproliferative diseases, myelodysplastic syndrome, and chronic myeloid leukemia (CML) were documented in the literature. Extramedullary recurrence of $\mathrm{CML}$ involving bone and forming granulocytic sarcoma is especially rare [1-3].

Alpha-fetoprotein (AFP) is a glycoprotein, which belongs to the albumin family and is mainly synthesized by fetal hepatocytes and yolk sacs. Increased AFP levels can be found in patients with reproductive system tumor, gastric cancer and lung cancer, especially primary hepatocellular carcinoma (PHC). AFP can be secreted by MS which is rare in report [4-7].

\section{Case Presentation}

Here we reported a rare MS case transformed from $C M L$, representing with multiple osteolytic damage, significant elevated AFP level as well as sequential ABL mutations during morphology and cytogenetic remission.

A 38-years-old man, who was diagnosed as CML chronic phase in 2012 and firstly treated with imatinib, was confirmed cytogenetics relapse after nine months. He was switched to dasatinib then achieved complete cytogenetic response and BCR-ABL transcript level was also lower than $0.01 \%$ three months later. From June 2017, the patient began to complain left buttock pain, subsequently pelvic and lumbar MRI revealed multiple abnormal signals and the following PET-CT showed extensive bone destruction as well as soft tissue mass, with abnormal FDG accumulation (Fig. 1A). Multiple myeloma was excluded by normal serum and urine immunofixation electrophoresis. To be mentioned, AFP level was found 
extremely high as $1191.30 \mathrm{ng} / \mathrm{mL}$ (reference value $<8.78 \mathrm{ng} / \mathrm{mL}$ ). Liver cancer has also been excluded by imageological examination. Left iliac mass biopsy showed the evidence of MS transformed from CML with AFP positive by immunohistochemical staining (Fig. 1B), strongly confirmed that AFP is secreted by MS, and bone marrow examination still showing chronic phase CML but with $0.13 \%$ BCR-ABL transcript level and F317L ABL mutation. Subsequently, combined chemotherapy (idarubicin and Cytarabine) was applied and dasatinib was replaced by nilotinib. His bone pain was gradually improved and AFP level was also going down to $200.32 \mathrm{ng} / \mathrm{mL}$. However, nine months later routine examination showed BCR-ABL was escalated to $6.4 \%$ again with AFP $1293.08 \mathrm{ng} / \mathrm{mL}$ (Fig. 1C). Next Generation Sequencing (NGS) was performed using repeated biopsy tissue as well as bone marrow and E255K mutation was also identified along with previous F317L mutation. In addition, EEPK1 and FBXW7 mutations were also identified but subsequently confirmed as germline mutation. Even though he received salvage combined chemotherapy, iliac mass was still significantly larger than before with the AFP peak level $12172.67 \mathrm{ng} / \mathrm{ml}$. The patient's condition was worsened rapidly and finally he was dead.

\section{Discussion And Conclusions}

To our knowledge, this case is the first report about MS with aberrant AFP expression. We confirmed AFP is produced by MS transformed from CML and its level is fluctuated with treatment outcome. In addition, bone marrow examination still showed CML CP but with molecular relapse and sequential $A B L$ mutation. Salvage chemotherapy combined with alternative tyrosine kinase inhibitor cannot rescue his worsening disease.

\section{Abbreviations}

MS, CML, AFP, PHC, NGS

Fig1: A: Lumbar and pelvic MRI showed multiple osteolytic damage and soft tissue mass. B: "left iliac mass" biopsy showed AFP positive by immunohistochemical staining. C: AFP level was found to increase with MS progression and decrease with remission, also be consistence with BCR-ABL level.

\section{Declarations}

Ethics approval and consent to participate: Not Applicable

Consent for publication: Consent for publication has been obtained from that person

Availability of data and materials: Not Applicable

Competing interests: The authors declare that they have no competing interests

Funding: The design of this case study and collection, analysis, and interpretation of the patient's data and in manuscript writing was supported by funds for key disciplines of Ruijin Hospital 
Authors' contributions: YW and SZ analyzed and interpreted the patient data regarding the rare hematological disease. HS prepared figure1 and was a major contributor in writing the manuscript. All authors read and approved the final manuscript.

Acknowledgements: Not Applicable

Authors' information (optional): Not Applicable

\section{References}

1. Arber DA, Orazi A, Hasserjian R, Thiele J, Borowitz MJ, Le Beau MM, et al. The 2016 revision to the World Health Organization classification of myeloid neoplasms and acute leukemia. Blood. 2016; 127:2391-405.

2. Almond LM, Charalampakis M, Ford SJ, Gourevitch D, Desai A. Myeloid sarcoma: presentation, diagnosis, and treatment. Clin Lymphoma Myeloma Leuk. 2017; 17:263-7.

3. Shahin OA, Ravandi F. Myeloid sarcoma. Curr Opin Hematol. 2017; 27:88-94.

4. Gillespie JR, Uversky VN. Structure and function of alphafetoprotein: a biophysical overview. Biochim Biophys Acta. 2000; 1480:41-56.

5. Taketa K. Alpha-fetoprotein: re-evaluation in hepatology. Hepatology. 1990; 12:1420-32.

6. Nomura F, Ohnishi K, Tanabe Y. Clinical features and prognosis of hepatocellular carcinoma with reference to serum alpha-fetoprotein levels: Analysis of 606 patients. Cancer. 1989; 64:1700-7.

7. Chan SL, Mo FK, Johnson PJ, Hui EP, Ma BB, Ho WM, et al. New utility of an old marker: Serial alphafetoprotein measurement in predicting radiologic response and survival of patients with hepatocellular carcinoma undergoing systemic chemotherapy. J Clin Oncol. 2009; 27:446-52.

\section{Figures}



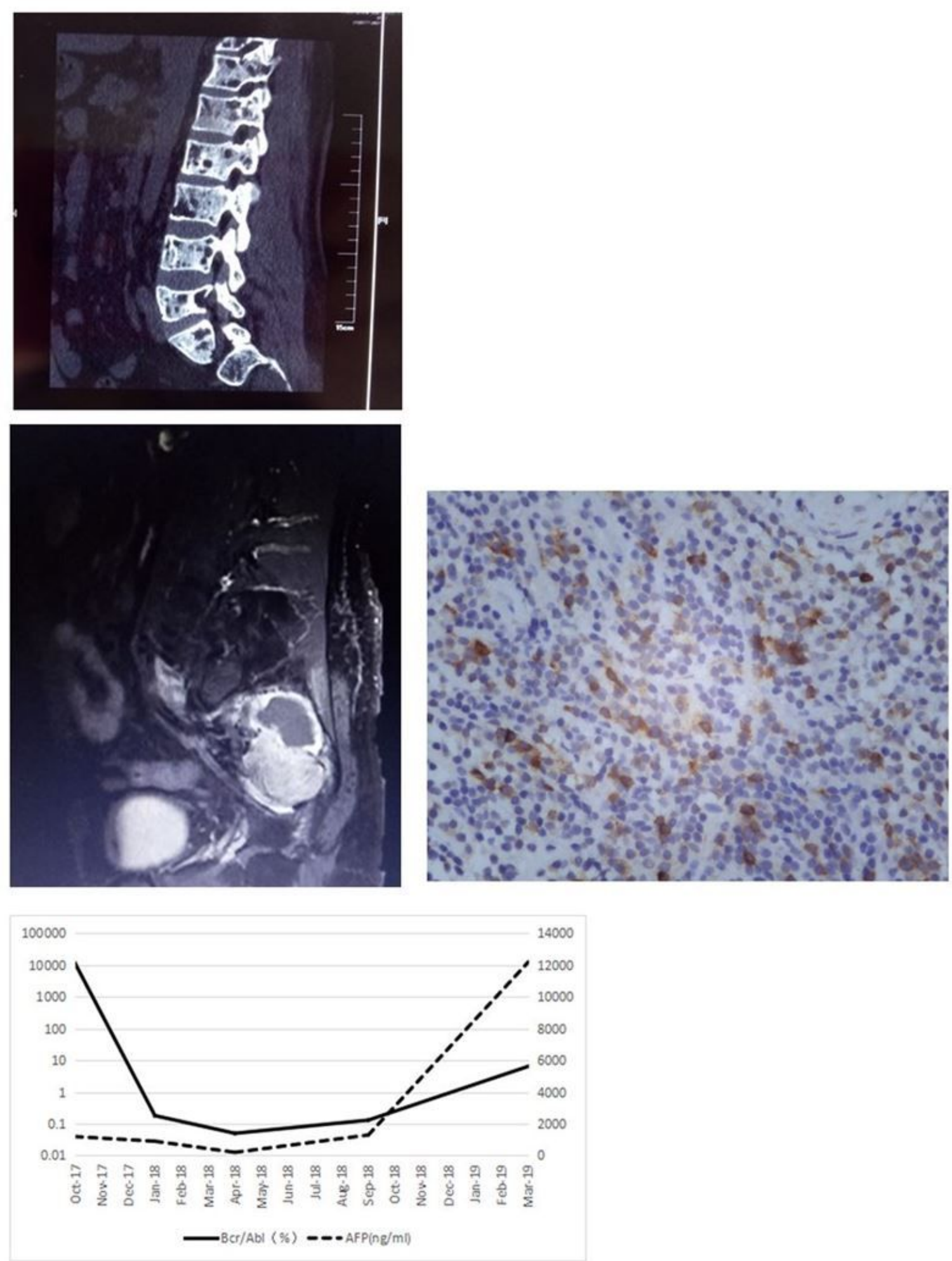

\section{Figure 1}

A: Lumbar and pelvic MRI showed multiple osteolytic damage and soft tissue mass.

B: "left iliac mass" biopsy showed AFP positive by immunohistochemical staining. 
C: AFP level was found to increase with MS progression and decrease with remission, also be consistence with BCR-ABL level. 